\title{
UV STABILITY OF HIGH BIREFIRNGENCE LIQUID CRYSTALS
}

\author{
Pao-Tai Lin and Shin-Tson Wu \\ School of Optics/CREOL, University of Central Florida, Orlando, \\ Florida 32816, USA \\ Chin-Yen Chang and Chain-Shu Hsu \\ Department of Applied Chemistry, National Chiao Tung University, \\ Hsinchu, Taiwan

\begin{abstract}
The ultraviolet stability of several high birefringence liquid crystal structures was studied. Compounds containing carbon-carbon double bond or triple bond are less stable than those containing merely phenyl rings. In addition to the structural effect, absorption wavelength also plays an important role to the degradation mechanisms.
\end{abstract}

Keywords: absorption; high birefringence; liquid crystals; photostability; structural effect

\section{INTRODUCTION}

High birefringence $(\Delta \mathrm{n} \geq 0.4)$ liquid crystals (LCs) are desirable for cholesteric displays, laser beam steering and optical switches for telecommunications [1]. A major concern for the high birefringence LCs is their ultraviolet (UV) stability. As the conjugation length increases, the LC absorption tail shifts toward $\lambda=365 \mathrm{~nm}$, the major UV light used for curing sealant. The material degradation is believed to originate from the UV-induced free radicals which, in turn, trigger chemical reactions with neighboring molecules and then disturb the LC alignment. When a LC cell is deteriorated by the accumulated UV exposure, the threshold voltage is smeared, birefringence decreased, viscosity increased and light scattering intensified [2].

This work was supported by AFOSR under contract number F49620-01-1-0377. The authors are indebted to Profs. R. Dabrowski of Military University of Technology, Warsaw (Poland), and L. C. Chien, A. Seed and M. Neubert of Kent State University for providing us the LC samples.

Address correspondence to Shin-Tson Wu, School of Optics/CREOL, University of Central Florida, Orlando, Florida 32816, USA. 
To enhance molecular conjugation, most high birefringence liquid crystals are composed of at least two phenyl rings or the combination of phenyl rings, acetylene (carbon-carbon triple bond) and stilbene (double bond) linking groups. The effective electronic conjugation of acetylene and stilbene is comparable to that of a phenyl ring. For example, tolane (phenyl-triple bond-phenyl) should have a similar conjugation length (or birefringence) to terphenyl, but its viscosity is lower than that of terphenyl because tolane has a smaller moment of inertia and more linear molecular shape. However, from UV stability viewpoint the phenyl ring system is more stable than the acetylene and stilbene.

In this paper, we have evaluated the UV stability of several high birefringence LC structures, including diphenyl-diacetylenes, tolanes, stilbenes and bistolanes. Our experimental results indicate that the UV degradation not only depends on the absorption wavelength but also depends on the detailed molecular constituents. Thus, a lower birefringence (i.e., shorter absorption wavelength) LC does not guarantee to have a better UV stability. The molecular composition is also an important factor.

\section{EXPERIMENT}

Owing to the elongated structure, most high birefringence LCs are solid at room temperature. In order to study the material stability at room temperature, unless specifically mentioned, we mixed $20 \%$ of the guest compound in a UV transparent nematic LC host MLC-6815. Homogeneously aligned cells were used for characterizing the phase retardation before and after UV exposure. The buffed polyimide alignment layer was found to help shield some UV light. As a result, such LC cell is more resistant to UV irradiation as compared to the $\mathrm{SiO}_{2}$ alignment layer. The peak wavelength of the UV lamp (Loctite) is $\lambda \sim 365 \mathrm{~nm}$. In all the data reported here, the UV intensity is fixed at $\mathrm{I}=250 \mathrm{mw} / \mathrm{cm}^{2}$ whereas the exposure time is accumulated. The UV light was incident to the LC cell at $\sim 10^{\circ}$ off normal. Its beam diameter covers the entire LC cell. To access the UV-induced LC degradation, a He-Ne laser beam $(\lambda=633 \mathrm{~nm})$ was used to measure the voltage-dependent transmittance under crossed polarizers. These data were then used to calculate the remaining phase retardation of the LC media. Based on these results, the degree of degradation can be assessed. Of course, phase change is not the only way for assessing the material degradation. As mentioned earlier, when degradation occurs threshold voltage is decreased simultaneously.

In order to correlate the degradation mechanism with absorption wavelength, the UV absorption of the compounds investigated were measured from $180-400 \mathrm{~nm}$. In these absorption experiments, $1 \%$ of the 


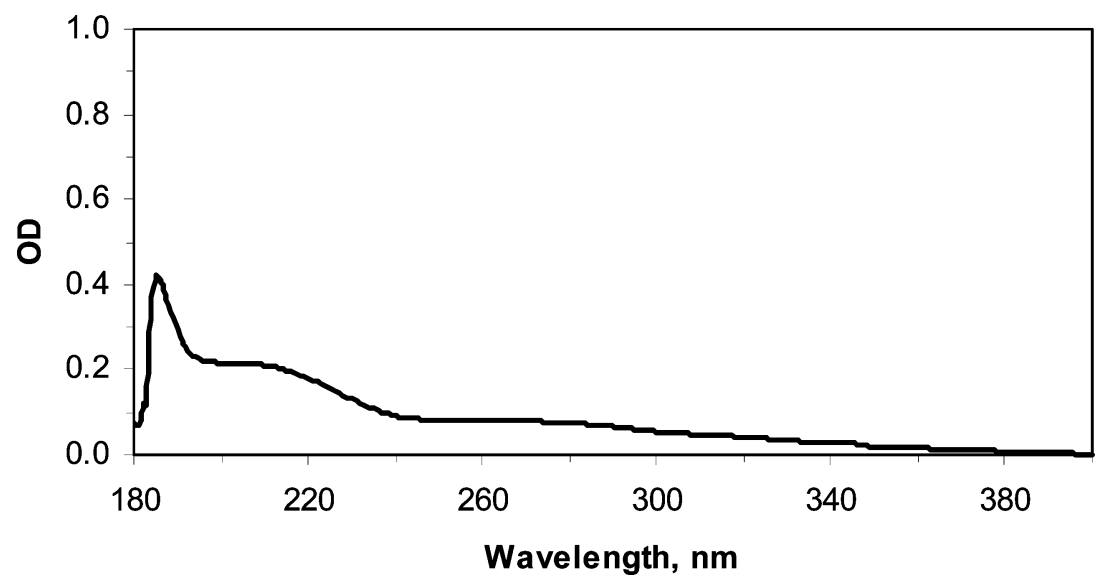

FIGURE 1 The measured UV absorption (optical density) of MLC-6815. A homogeneous cell with quartz substrates without ITO and $6 \mu \mathrm{m}$ gap was used for this measurement.

high birefringence compound under investigation was dissolved in the Merck MLC-6815. The absorption spectrum of MLC-6815 was measured using a homogeneous quartz cell without indium-tin-oxide coating. The cell gap is $6 \mu \mathrm{m}$. Results are shown in Figure 1. From Figure 1, MLC-6518 is highly transparent in the UV region. A small absorption near $190 \mathrm{~nm}$ region occurs. For measuring the UV absorption of high birefringence compounds, we used the double cell method. Two $6 \mu \mathrm{m}$ quartz cells (one served as sample and another as reference) without indium-tin-oxide coating were used. A computer-controlled dual channel Cary-500 spectrophotometer was used as light source.

Table 1 lists the ten liquid crystal compounds and mixtures used for the UV studies. For simplicity, we use the following abbreviations: diphenyl-diacetylenes (PTTP), tolanes (PTP), isothiocyanato naphthalene tolanes (NTP), bistolane (PTPTP), cyclehexane-stilbene (CPDP), and biphenyl-tolane (PPTP). In the above notations, $\mathrm{P}$ stands for phenyl ring, $\mathrm{T}$ for carbon-carbon triple bond, $\mathrm{N}$ for naphthalene, $\mathrm{C}$ for cyclohexane ring and D for carbon-carbon double bond. A Merck mixture E7 was also included for comparison.

\section{RESULTS AND DISCUSSIONS}

\subsection{Diphenyl-diacetylenes (PTTP)}

Diphenyl-diacetylene liquid crystals exhibit a high birefringence, low viscosity and wide nematic range except that its UV and thermal stability 
TABLE 1 The Molecular Structures and Phase Transitions Temperatures $\left({ }^{\circ} \mathrm{C}\right)$ of the LC Compounds and Mixtures Used for the UV Stability Measurements. K, N, Sm, I and () Represent Crystalline, Nematic, Smectic, Isotropic and Monotropic Phase, Respectively

(n)

is inadequate [3-6]. The binary mixture consisting of $50 \mathrm{wt} \%$ PTTP-24 and $50 \mathrm{wt} \%$ PTTP-36 (\#1 in Table 1) can only resist UV irradiation (at $\mathrm{I}=250 \mathrm{mw} / \mathrm{cm}^{2}$ ) for $\sim 10$ seconds.

Figure 2 shows the voltage-dependent transmittance curves for the fresh sample and after UV exposure for 65 seconds at $\mathrm{I}=50 \mathrm{mw} / \mathrm{cm}^{2}$. From Figure 2, three general phenomena are observed as degradation takes place: 1 . the effective $\Delta \mathrm{n}$ is decreased, 2 . the threshold voltage is smeared and decreased, and 3. the light scattering is gradually intensified. Since the absorption tail of PTTP is below $365 \mathrm{~nm}$, the degradation is primarily 


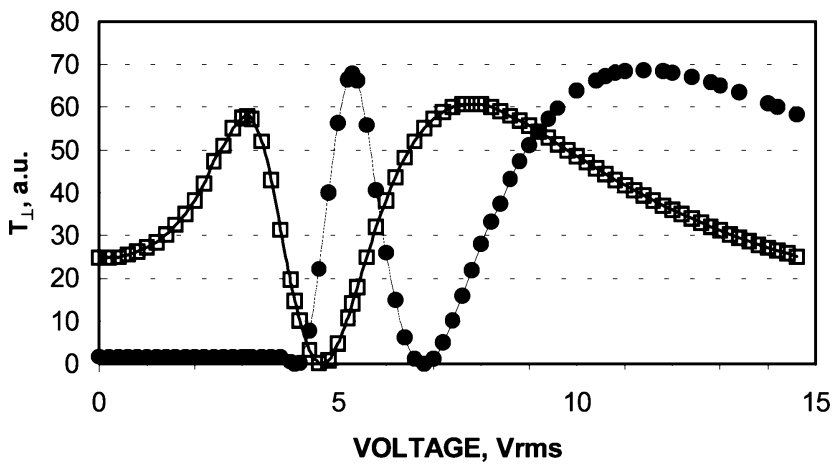

FIGURE 2 The voltage-dependent transmittance of a homogeneous LC cell before (dots) and after (squares) UV exposure. UV intensity I $=50 \mathrm{mw} / \mathrm{cm}^{2}$; exposure time $=65$ seconds. LC: PTTP-24/36 (structure $\# 1$ ), cell gap $\mathrm{d}=3.74 \mu \mathrm{m}$ and $\mathrm{T}=22^{\circ} \mathrm{C}$. Alignment layer is a buffed polyimide film. Crossed polarizers. $\lambda=633 \mathrm{~nm}$.

caused by the structural effect rather than absorption. The degradation mechanism is believed to originate from the UV-induced free radicals of the diacetylene group. These free radicals transfer charges to the neighboring PTTP molecules, converting their triple bonds into double bonds, and initiating the polymerization process from front surface layers and gradually migrating into bulk as UV dosage increases [2]. Once the surface layers are cross-linked, the surface alignment is disturbed resulting in a reduced $\Delta \mathrm{n}$, increased viscosity, smeared threshold voltage, and increased light scattering.

\subsection{Bistolane, Naphthalene Tolane and Biphenyl-tolane}

The molecular structures of the bistolane [7-9], naphthalene tolane [10], and biphenyl tolane [11,12] we studied are listed as \#2-4 in Table 1 and their nematic range is $73.5 \rightarrow 150.5, \quad 95.3 \rightarrow 135.2$ and $185.7 \rightarrow 212^{\circ} \mathrm{C}$, respectively. These compounds have birefringence in the 0.35 to 0.55 range. Their UV stability and absorption spectra are shown in Figures 3 and 4 , respectively.

From Figure 3, their UV stability has following sequence: biphenyl tolane (structure \#4) > naphthalene $(\# 3)>$ bistolane $(\# 2)$. As shown in Figure 4, naphthalene has the longest absorption tail, followed by bistolane and then biphenyl tolane. Although naphthalene has longer absorption than bistolane, its UV stability is much better than that of bistolane. This indicates that absorption is not the only critical factor affecting the UV 


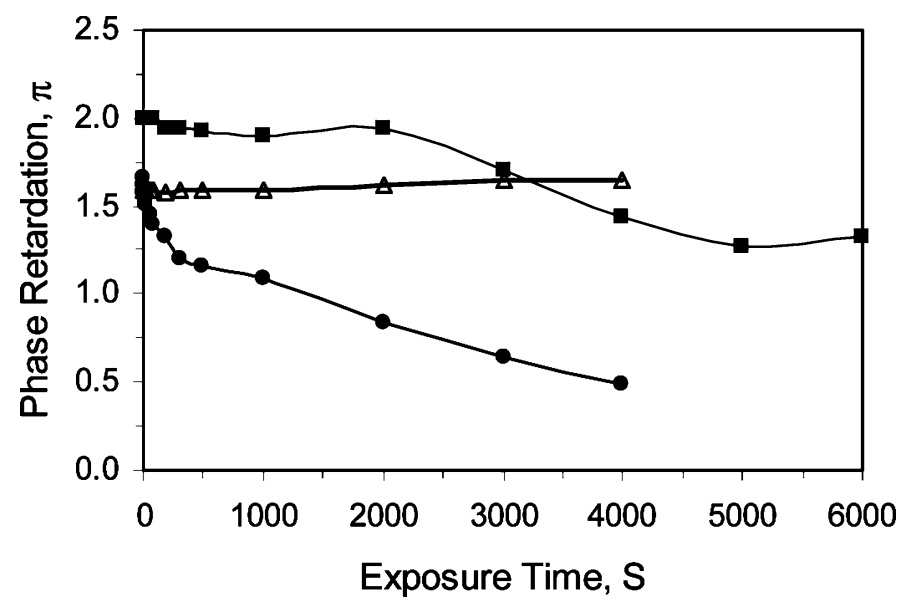

FIGURE 3 The exposure time dependent phase retardation of the bistolane, naphthalene tolane and biphenyl-tolane LC cells. Dots: fluoro bistolane (structure \#2), squares: NCS naphthalene tolane (structure \#3), and triangles: biphenyltolane (structure \#4). Guest LC concentration: 20\%. Host LC: MLC-6815. UV intensity $=250 \mathrm{mw} / \mathrm{cm}^{2}$. Cell gap $\mathrm{d} \sim 4 \mu \mathrm{m} . \lambda=633 \mathrm{~nm}$.

stability. Molecular constituents are also important. Naphthalene contains only one triple bond and bistolane has two. The triple bond is believed to be the weakest part within these compounds.

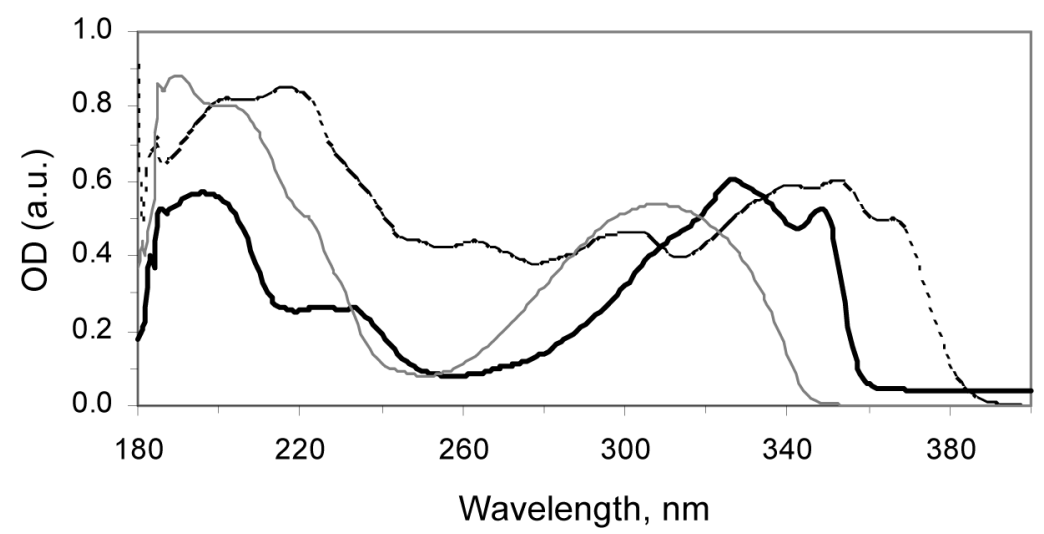

FIGURE 4 The UV absorption spectra (optical density) of fluoro bistolane (solid lines), NCS naphthalene tolane (dashed lines) and fluoro biphenyl-tolane (gray lines). Guest LC concentration: 1\%. Host LC: MLC-6815. Cell gap d $=6 \mu \mathrm{m}$. 
As compared to the PTTP series, bistolane's UV stability is about $10 \times$ better. Although bistolane and diphenyl-diacetylene both have two acetylene groups, the acetylene groups in bistolane are separated by a phenyl ring. Finally, biphenyl tolane exhibits an excellent stability. No degradation was observed up to 4000 seconds for the $20 \%$ mixture employed. This remarkable result could be explained that the PPTP-3F compound has little absorption at $\lambda=365 \mathrm{~nm}$, as shown in Figure 4.

\subsection{Cyclohexane Stilbene}

Two cyclohexane stilbenes were studied. Their molecular structures are shown as \#5 and 6 in Table 1. The UV stability and absorption spectra of stilbene (\#5) and $\alpha$-methyl stilbene (\#6) are shown in Figures 5 and 6 , respectively. Although these stilbene compounds have negligible absorption at $\lambda=365 \mathrm{~nm}$, their UV stability is inadequate. The double bond can be easily polymerized under UV exposure. The $\alpha$-methyl homologue slightly retards the polymerization process, however, its stability is still far from satisfaction. Although stilbene has a longer effective conjugation than acetylene, its UV stability is worse. Thus, from UV stability viewpoint, double bond is not a favored choice for extending molecular conjugation while designing high birefringence LC compounds.

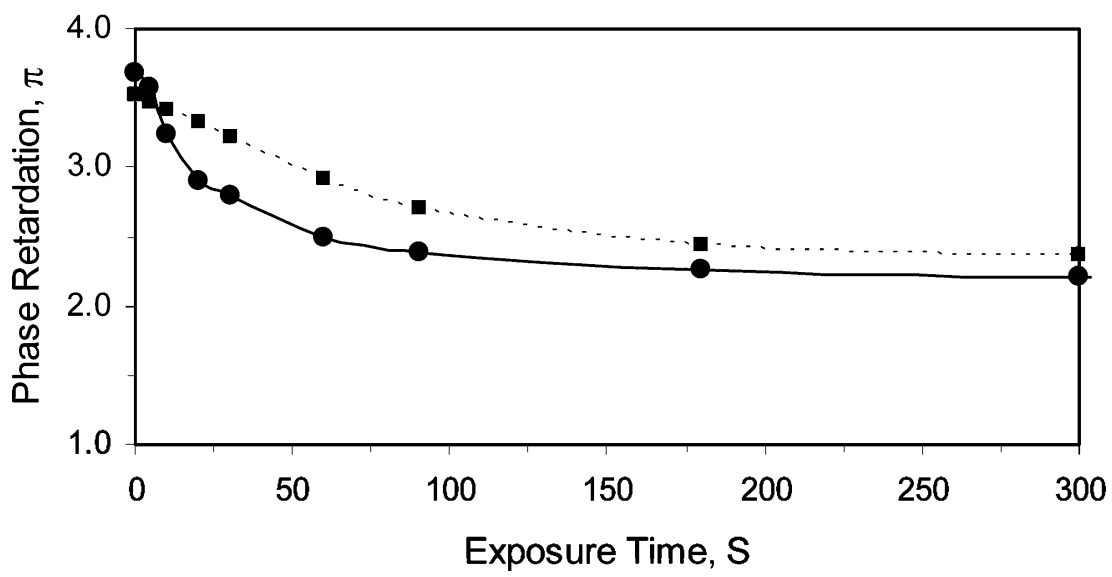

FIGURE 5 The exposure time dependent phase retardation of stilbene and $\alpha$-methyl stilbene LC cells. Dots: structure \#5, squares: structure \#6. Guest LC concentration: $20 \%$. Host LC: MLC-6815. UV intensity $=250 \mathrm{mw} / \mathrm{cm}^{2}$. Cell gap $\mathrm{d}=10 \mu \mathrm{m} . \lambda=633 \mathrm{~nm}$. 


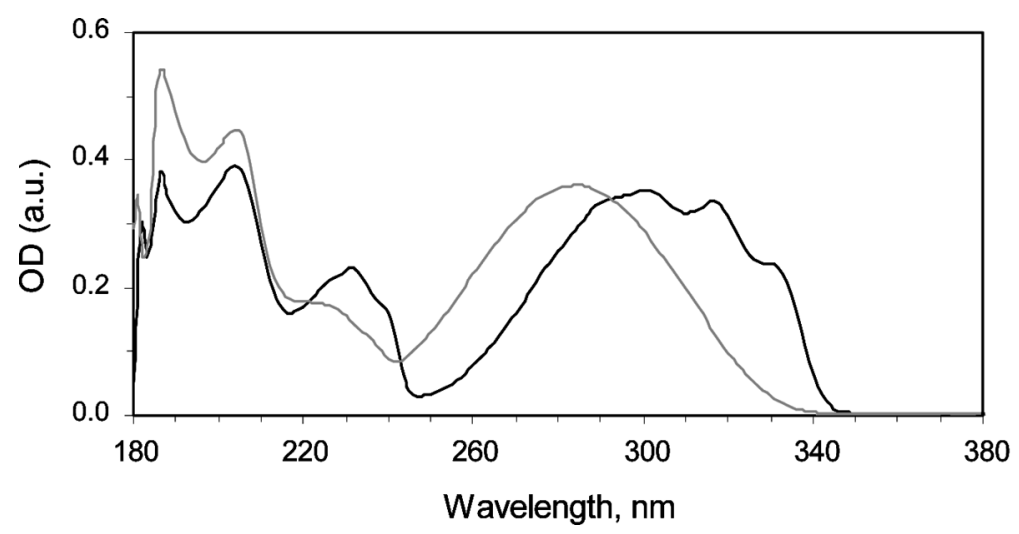

FIGURE 6 The UV absorption spectra (optical density) of stilbene (black lines) and $\alpha$-methyl stilbene (gray lines). Guest LC concentration: $1 \%$. Host LC: MLC-6815. Cell gap $\mathrm{d}=6 \mu \mathrm{m}$.

\subsection{Tolanes (PTP)}

The isothiocyanato (NCS) tolanes exhibit a relatively high birefringence, large dielectric anisotropy and low viscosity. The major problem remains to be overcome is to discover more such compounds having a wide nematic range.

The UV stability and absorption of the alkyl, alkoxy and difluoro NCS tolanes were studied. Their structures are shown as \#7, 8 and 9 in Table 1, respectively. The pentyl NCS tolane (\#7) exhibits a monotropic smectic phase with melting point at $91.2^{\circ} \mathrm{C}$, the alkoxy NCS tolane has a pure smectic E phase from 83.1 to $113.8^{\circ} \mathrm{C}$ and the difluoro NCS tolane has an enantiotropic nematic phase from 49.5 to $56.6^{\circ} \mathrm{C}$. Their UV stability and absorption spectra are depicted in Figures 7 and 8, respectively.

From Figure 7, the UV stability of this NCS tolane series follows the order: $\# 8>\# 7>\# 9$. The $20 \%$ alkoxy NCS tolane mixture could withstand $\sim 3000$ seconds of UV irradiation before a noticeable degradation takes place. For the alkyl and difluoro NCS tolanes, their lifetime is reduced to 2000 and 1000 seconds, respectively.

From the absorption curves shown in Figure 8, the alkoxy difluoro NCS tolane (\#9) has the longest absorption wavelength, followed by alkoxy NCS tolane (\#8) and then alkyl NCS tolane (\#7). Since the absorption tail of the alkoxy difluoro NCS tolane extends to $\lambda=365 \mathrm{~nm}$ (the major UV lamp wavelength), its stability is the poorest among the three similar structures investigated. However, between the alkyl and alkoxy side chains, the alkoxy homologue exhibits a better stability than the alkyl one although its absorption is somewhat longer. 


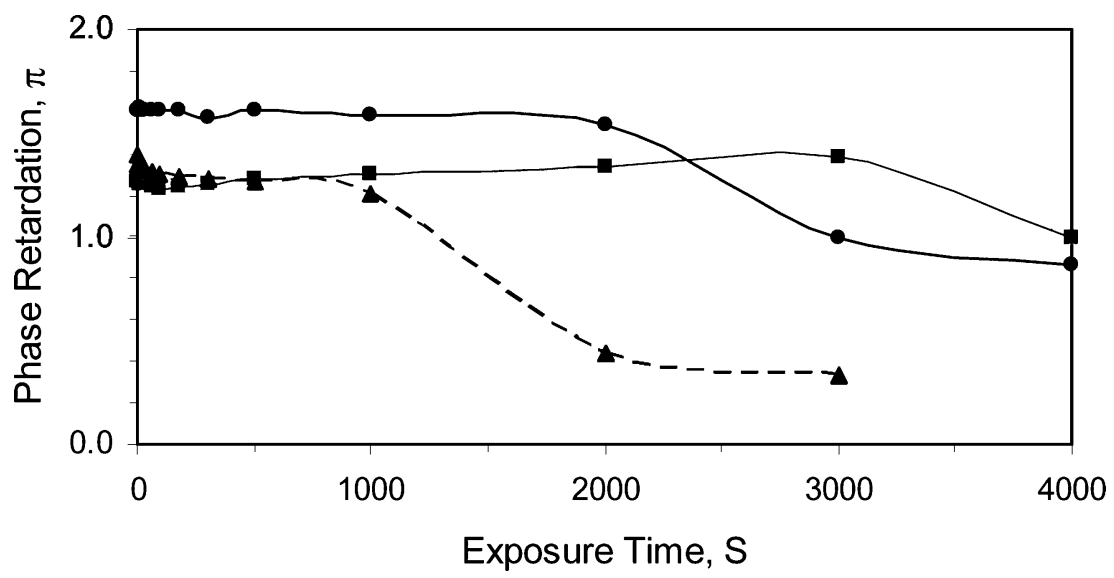

FIGURE 7 The exposure time dependent phase retardation of three NCS tolane LC cells. Dots: alkyl NCS tolane (structure \#7), squares: alkoxy NCS tolane (structure \#8) and triangles: difluoro alkoxy NCS tolane (structure \#9). Guest LC concentration: $20 \%$. Host LC: MLC-6815. UV intensity $=250 \mathrm{mw} / \mathrm{cm}^{2}$. Cell gap $\mathrm{d}=4 \mu \mathrm{m}$. $\lambda=633 \mathrm{~nm}$.

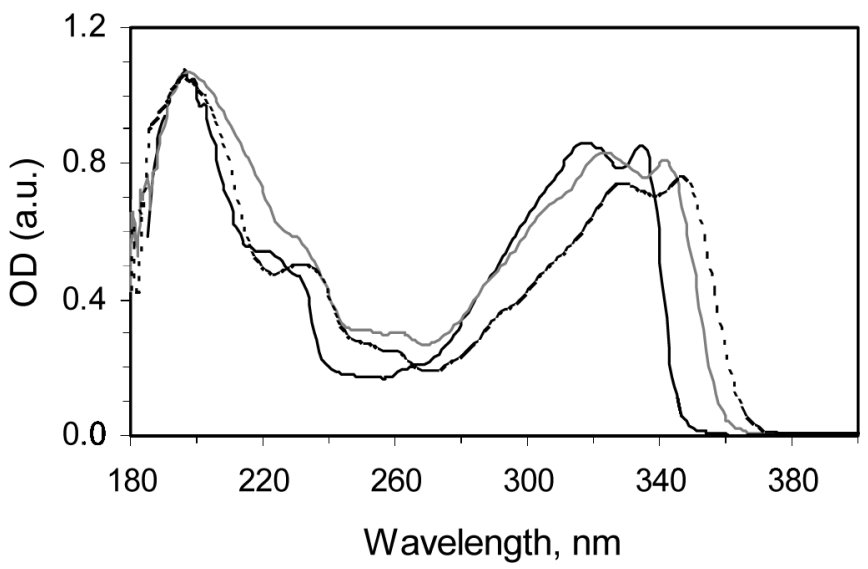

FIGURE 8 The UV absorption spectra (optical density) of three NCS tolane samples. Solid lines: alkyl NCS tolane (structure \#7), gray lines: alkoxy NCS tolane (structure \#8) and dashed lines: difluoro NCS tolane (structure \#9). Guest LC concentration: $1 \%$. Host LC: MLC-6815. Cell gap $\mathrm{d}=6 \mu \mathrm{m}$. 


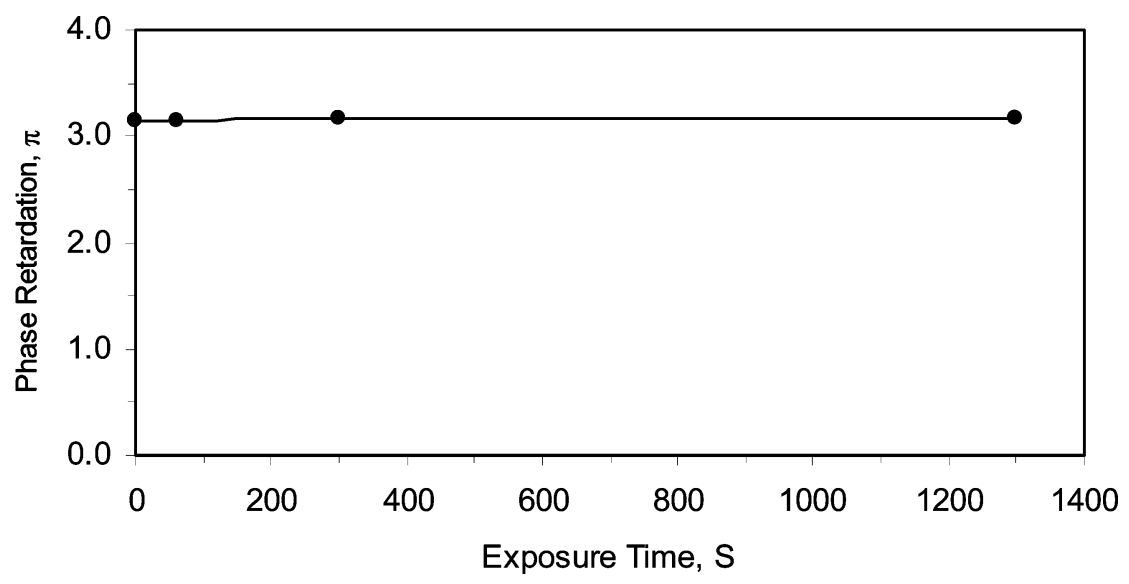

FIGURE 9 The exposure time dependent phase retardation of E7 LC mixture. UV intensity $=250 \mathrm{mw} / \mathrm{cm}^{2}$. Cell gap $\mathrm{d} \sim 4 \mu \mathrm{m} . \lambda=633 \mathrm{~nm}$.

\subsection{E7 Mixture}

For the comparison purpose, we also evaluated a Merck LC mixture E7 which has been used in the optical phased arrays for laser beam steering [13]. E7 consists of four components: 5CB (4'-pentyl-4-cyanobiphenyl), $7 \mathrm{CB}, 5 \mathrm{OCB}$ and 5CT (cyano-terphenyl). Its birefringence is 0.22 at

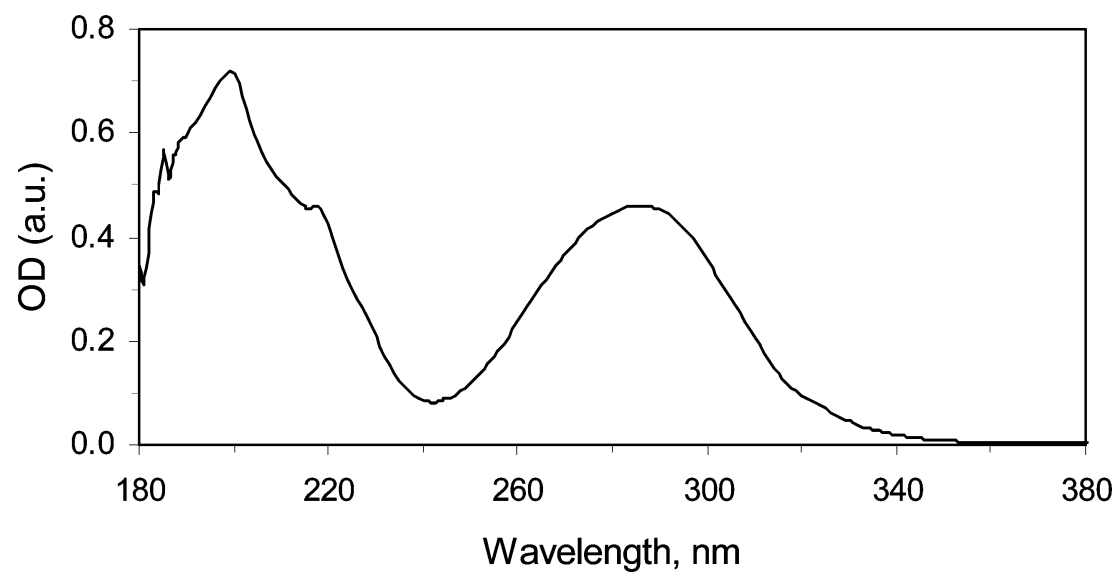

FIGURE 10 The UV absorption spectra (optical density) of E7 LC mixture. E7 concentration: $1 \%$. Host LC: MLC-6815. Cell gap $d=6 \mu \mathrm{m}$. 
$\lambda=633 \mathrm{~nm}$ and $\mathrm{T} \sim 22^{\circ} \mathrm{C}$. Figures 9 and 10 plot the UV stability and absorption spectra of E7. It should be mentioned that E7 is liquid at room temperature so that its UV stability measurement was conducted using $100 \%$ E7; not 20\% dissolved in the MLC-6815 host. From Figure 9, up to 1300 seconds of exposure at $\mathrm{I}=250 \mathrm{mw} / \mathrm{cm}^{2}$ no sign of degradation is observed. From Figure 10, the absorption tail of E7 is below $350 \mathrm{~nm}$. Thus, E7 has little absorption at $\lambda=365 \mathrm{~nm}$. This is one of the reasons that $\mathrm{E} 7 \mathrm{has}$ such a long lifetime. Another important reason is that all the E7 constituents contain no triple bond or double bond. Both biphenyl and terphenyl are quite resistant to UV exposure. This information is important for designing new generation high birefringence LC compounds.

\section{CONCLUSIONS}

Molecular structure and absorption play important roles in governing the UV stability of high birefringence liquid crystals. Double bond and carbon-carbon triple bond could initiate the polymerization process and then disturb the LC alignment. As a result, the LC threshold voltage and effective birefringence are decreased and light scattering intensified. On the other hand, biphenyl and terphenyl are much more stable than the acetylene group except that their viscosity is higher. How to design high birefringence liquid crystals while possessing low viscosity and long lifetime is a challenging task.

\section{REFERENCES}

[1] Wu, S. T. \& Yang, D. K. (2001). Reflective Liquid Crystal Displays, Wiley: New York.

[2] Wu, S. T. (2001). SID Tech. Digest, 32, 954.

[3] Grant, B. (1978). Mol. Cryst. Liq. Cryst., 48, 175.

[4] Wand, M. D., Vohra, R. T., Thurmes, W. N., \& More, K. M. (1996). Ferroelectrics, 180, 333.

[5] Wu, S. T., Margerum, J. D., Meng, B. H., Dalton, L. R., Hsu, C. S., \& Lung, S. H. (1992). Appl. Phys. Lett., 61, 630.

[6] Wu, S. T. \& Neubert, M. (2001). U.S. patent 6, 312, 618.

[7] Goto, Y. \& Kitato, K. (1989). EP 345, 013.

[8] Sekine, C., Fujisawa, K., Fujimoto, Y., \& Minai, M. (1999). Mol. Cryst. Liq. Cryst., 332, 235.

[9] Wu, S. T., Neubert, M. E., Keast, S. S., Abdallah, D. G., Lee, S. N., Walsh, M. E., \& Dorschner, T. A. (2000). Appl. Phys. Lett., 77, 957.

[10] Seed, A. J., Toyne, K. J., Goodby, J. W., \& Hird, M. (2000). J. Mater. Chem., 10, 2069.

[11] Takatsu, H., Tanaka, Y., \& Sato, H. (1988). U. S. Patent 4,726, 910.

[12] Hirschmann, H. \& Reiffenrath, V. (2000). 18th Int'l Liq. Cryst. Conf., Sendai: Japan.

[13] McManamon, P. F., Dorschner, T. A., Corkum, D. L., Friedman, L., Hobbs, D. S., Holz, M., Liberman, S., Nguyen, H. Q., Resler, D. P., Sharp, R. C., \& Watson, E. A. (1996). Proc. IEEE, 84, 268. 